\title{
Von Willebrand factor and coagulation factor VIII in Moyamoya disease associated with Graves' disease: A case report
}

\author{
SHOU-CHEN REN ${ }^{1}$, BAO-QIN GAO $^{1}$, WEI-LI YANG ${ }^{1}$, WEI-XIN FENG ${ }^{1}$, \\ JIAN XU ${ }^{2}$, SHAO-WU LI ${ }^{3}$ and YONG-JUN WANG ${ }^{4}$ \\ Departments of ${ }^{1}$ Pediatrics and ${ }^{2}$ Endocrinology, Beijing Tian Tan Hospital; ${ }^{3}$ Department of Functional Neuroimaging, \\ Beijing Neurosurgical Institute; ${ }^{4}$ Department of Neurology, Beijing Tian Tan Hospital, \\ Capital Medical University, Beijing 100050, P.R. China
}

Received July 28, 2015; Accepted September 9, 2016

DOI: 10.3892/etm.2016.3769

\begin{abstract}
The present study reported the case of a Chinese boy who was diagnosed with Moyamoya disease (MMD) associated with Graves' disease (GD). An overactivation of von Willebrand factor (vWF) and coagulation factor VIII (FVIII) was identified in the plasma of the patient. Thiamazole and metoprolol treatment was thus administrated. After 2 months of treatment, the patient's thyroid function returned to normal and the neurological symptoms improved gradually. At the same time, the activities of vWF and FVIII were depressed. During the 20-month follow-up, information regarding the neurological symptoms, cerebrovascular imaging, thyroid function, thyroid autoantibodies and coagulation parameters was collected. High levels of thyroid autoantibodies persisted throughout the follow-up period, while other coagulation parameters remained in the normal range. In conclusion, considering the vital role of vWF and FVIII in vascular diseases, it is hypothesized that these two factors may serve an important role in the occurrence of GD associated with MMD.
\end{abstract}

\section{Introduction}

Moyamoya disease (MMD) is a progressive cerebrovascular steno-occlusive disorder. It typically involves the distal internal carotid arteries (ICAs), the proximal anterior cerebral arteries (ACAs) and the middle cerebral arteries (MCAs), accompanied by the development of an abnormal collateral vascular network at the base of the brain (1-3). Thus far, the majority of MMD cases have been reported in Eastern Asia (4); however, even in that region, the coexistence of MMD and Graves' disease (GD) is rare. In 1991, Kushima et al first reported two cases

Correspondence to: Dr Yong-Jun Wang, Department of Neurology, Beijing Tian Tan Hospital, Capital Medical University, 6 Tian-Tan Xi Li, Dongcheng, Beijing 100050, P.R. China

E-mail: bouyren@126.com

Key words: Moyamoya disease, Graves' disease, von Willebrand factor, coagulation factor VIII of females suffering from MMD and GD simultaneously (5). To the best of our knowledge, only $~ 50$ cases of simultaneous MMD and GD have been described in the literature available in the MEDLINE database to date. The patients were predominantly women, and their ages ranged between 10 and 54 years (mean, 29.3 years). Transient ischemic attacks and cerebral infarction were the most common symptoms in these patients. The majority of the cases showed thyrotoxicity when the cerebral ischemic event occurred and eventually recovered from the neurological symptoms after medical and/or surgical treatment (6). Although the association between MMD and GD remains unclear, a number of previous studies have suggested that GD could induce the development of MMD through 'thyroid storm' $(7,8)$, 'cerebrovascular hemodynamic changes' (9) and 'autoimmune arteritis' (10-13).

Von Willebrand factor (vWF) is a useful marker of endothelial activation or dysfunction (14). Increasing evidence has demonstrated that vWF is involved in cardiovascular (15) and ischemic cerebrovascular diseases $(16,17)$. In addition, coagulation factor VIII (FVIII) is involved in the process of thrombosis, and elevated FVIII levels are a prothrombotic risk factor for thromboembolism (18). The correlation of hyperthyroidism with elevated FVIII and vWF activities has been previously reported (19).

The present study reported the case of a 12-year-old male with significant overactivation of vWF and FVIII. The patient suffered from the rare coexistence of GD with MMD. Thus, a comprehensive investigation of a potential correlation between GD and the overactivation of vWF/FVIII and MMD was performed. Written informed consent was obtained from the family.

\section{Case report}

A 12-year-old Chinese male was admitted to the Beijing Tian Tan Hospital (Beijing, China) in May 2013 with a rapidly progressive mild quadriplegia, aphasia and urinary incontinence. At 5 days prior to admission, the patient experienced several episodes of vomiting, paroxysmal aphasia and mild hemiplegia on his left side, along with recurrent brief convulsions. The condition of the patient rapidly deteriorated during the subsequent 5 days. The patient had previously suffered irritability, tremors, excessive sweating and frequent palpitations following moderate 
physical activity for approximately 1 year. He had no other disease history and a relevant family disease history.

On admission, the patient was dysphoric with slurred speech, and had a high blood pressure $(165 / 95 \mathrm{mmHg})$, and rapid and regular heart rate (110-140 bpm). In addition, the patient presented facial paralysis (skewing of the mouth to the right side) and mild quadriplegia with globally increased muscle tone. Hyperactive deep tendon reflexes and positive bilateral Babinski signs were observed. A moderately enlarged thyroid gland was also identified. Emergency head magnetic resonance imaging (MRI) scans revealed multiple regions of cerebral infarction that could not be attributed to a single vascular territory (Fig. 1). In addition, magnetic resonance angiography (MRA) revealed severe stenosis at the bilateral terminal portion of the ICAs, while the bilateral MCAs and ACAs had almost disappeared (Fig. 1). Accordingly, combined with the patient's symptom of progressive mild quadriplegia, aphasia, recurrent convulsions and urinary incontinence that appeared 5 days prior to the first admission, and the signs of globally increased muscle tone, hyperactive deep tendon reflexes and positive bilateral Babinski signs on admission and the results of the head MRI, a diagnosis of multiple cerebral infarction was made definitely. Blood examination showed the following results: Thyroid-stimulating hormone (TSH), $<0.001 \mu \mathrm{U} / \mathrm{ml}$ (reference range, 0.35-4.94 $\mu \mathrm{U} / \mathrm{ml}$ ); and free triiodothyronine (FT3), $29.17 \mathrm{pmol} / 1$ (reference range, 2.63-5.7 pmol/l). Positive thyrotropin receptor antibody (TR-Ab), as well as elevated levels of thyroid peroxidase antibody (TPO-Ab; $396.44 \mathrm{U} / \mathrm{ml}$; reference range, $<12 \mathrm{U} / \mathrm{ml}$ ) and thyroglobulin antibody (TG-Ab; $134.56 \mathrm{U} / \mathrm{ml}$; reference range, $<34 \mathrm{U} / \mathrm{ml}$ ), were also detected in the blood (Table I). Due to the above symptoms of irritability, tremors, excessive sweating, frequent palpitations and enlarged thyriod gland with elevated TPO-Ab, elevated TG-Ab and positive TR-Ab in blood, GD was suspected firstly. A thyroid gland needle biopsy revealed thyroid follicular epithelial cell proliferation, which confirmed the diagnosis of GD. In addition, quantification of coagulation factor activity demonstrated that FVIII activity was $261.6 \%$ (reference range, 50-150\%) and vWF activity was $324.2 \%$ (reference range, 40-120\%), while other coagulation parameters were all within the normal ranges, as shown in Table I.

The patient was managed with standard antithyroid treatment $(20 \mathrm{mg} /$ day thiamazole, Merck Serono, Darmstadt, Germany; $100 \mathrm{mg} /$ day metoprolol, AstraZeneca Co., Ltd., Wuxi, China) until the submission of this manuscript, in combination with a low iodine diet. In beginning of the treatment, other management included $30 \mathrm{mg} /$ day Adalat for 2 weeks (Bayer Schering Pharma AG) for control of blood pressure, sedative administration (5 mg diazepam, Zhejiang Medicine Co., Ltd., Zhejiang, China) lasting until the calming of irritability after 1 week. However, no anticoagulants or thrombolytic agents were administered to the patient. After 2 months of treatment, the neurological symptoms had improved significantly; the patient had recovered from paralysis, walked with a steady gait and no slurred speech was observed. Further blood tests showed that the patient's thyroid function had almost returned to normal (TSH, $0.027 \mu \mathrm{U} / \mathrm{ml}$; FT3, $3.21 \mathrm{pmol} / \mathrm{l})$, with depressed vWF and FVIII activity (117.10 and $122.40 \%$, respectively), although the thyroid autoantibodies persisted at high levels (Table I). Digital subtraction angiography (DSA) was then performed, which demonstrated severe stenosis of the bilateral distal segments of the ICAs, and of the ACAs and MCAs. In addition, only minimal antegrade flow of ACA and MCA territories was observed. A network of collateral vessels was also visible at the base of the brain and in the bilateral basal ganglia areas (Fig. 2). To prevent further attacks, revascularization surgery was performed initially on the right side superficial temporal artery binding to the middle meningeal artery at 3 months after the first admission. This results of DSA associated with the symptoms of gradually emerged quadriplegia, aphasia, convulsions and the head MRI results which showed bilateral basal ganglia and subcortical multiple infarction, together, could meet the diagnostic criteria for MMD (20). After a further 3 months (6 months after the first admission), an encephalo-duro-arterio-synangiosis procedure was performed successfully on the left side superficial temporal artery attaching to the endocranium and arachnoid.

During the 20-month follow-up period, a continuous $20 \mathrm{mg} /$ day thiamazole and $100 \mathrm{mg} /$ day metoprolol was administrated, the thyroid function, thyroid autoantibodies and coagulation parameters were measured every 3 months. During this period, a correlation was observed between the patient's thyroid function and vWF/FVIII activity. After 3 months, following the administration of propranolol and metoprolol, his thyroid function (FT3 and FT4) recovered to the normal range. Notably, a decline in FVIII/vWF activity was observed simultaneously. After a further 3 months, while FT3 and FT4 in the patient's blood were higher than the normal range, a homologous elevation of the patient's FVIII/vWF activity in the blood was observed. A similar phenomenon was observed during the next 12-month follow-up period (Table I). When the thyroid function recovered to the normal range, a corresponding decline in FVIII/vWF activity was observed (Table I). At the same time, the thyroid autoantibodies persisted at high levels, whereas other coagulation parameters were consistently around the normal ranges.

\section{Discussion}

The case reported in the present study was affected by GD and MMD successively. The manifestations observed were mainly due to cerebral infarction, while the clinical features of thyrotoxicosis had appeared approximately 1 year prior to admission. This is consistent with the features of previously reported cases, which suggest that transient ischemic attacks and cerebral infarction were the common symptoms and thyrotoxicity was showed when the cerebral ischemic event occurred (6).

Due to the limited incidence of co-existing GD and MMD, extensive studies have not been possible, and no specific mechanism underlying this association has been elucidated to date. Previous hypotheses on the underlying mechanism have been proposed that presume the existence of a pathoetiological association between GD and MMD, such as genetic background, autoimmunization $(10,11)$, thyrotoxic states $(9,21)$ and significant hemodynamic changes (9). There are certain evidence-based medicine practices and epidemiological data that support these theories. For instance, Kim et al concluded that elevated thyroid autoantibodies are associated with MMD, based on a prospective study (11). This theory was supported 
A

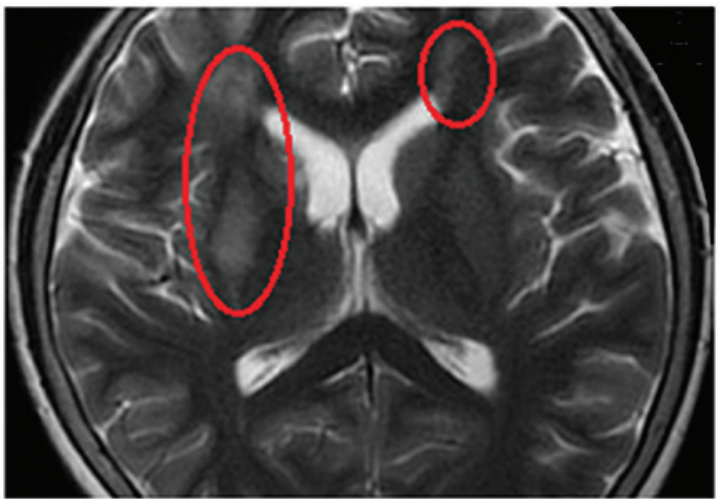

C

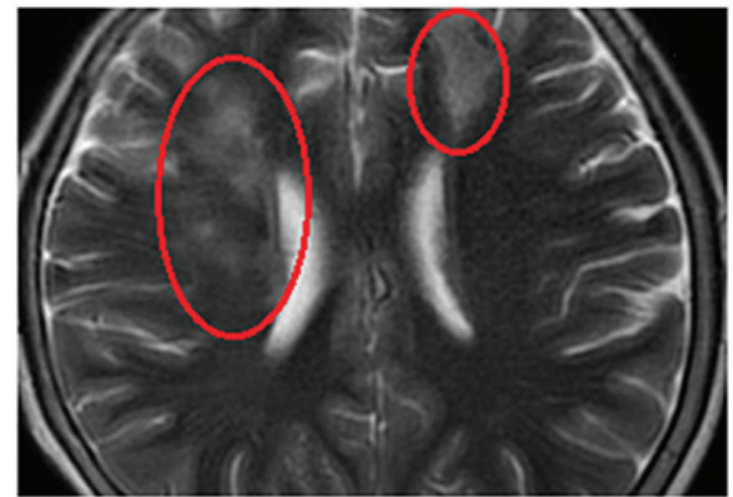

E

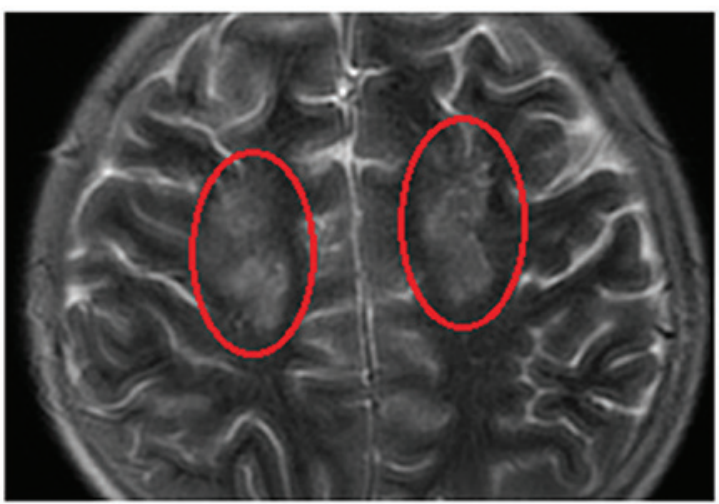

$\mathbf{G}$

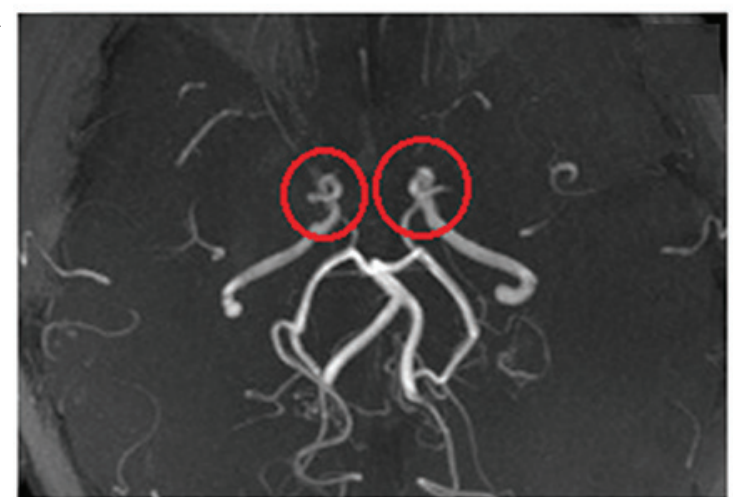

B

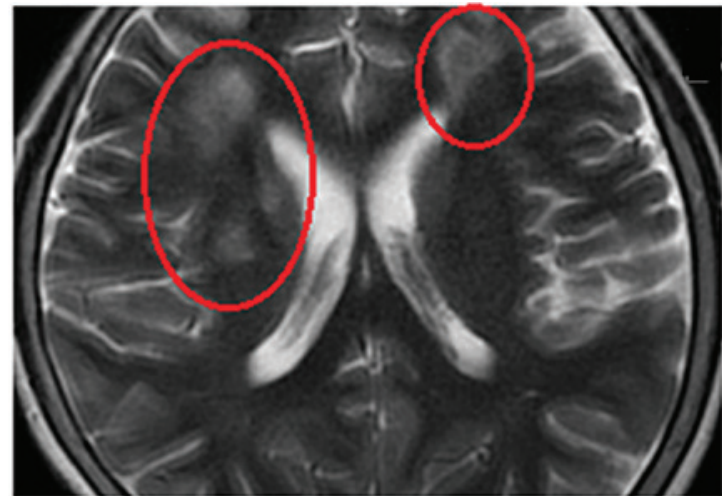

D

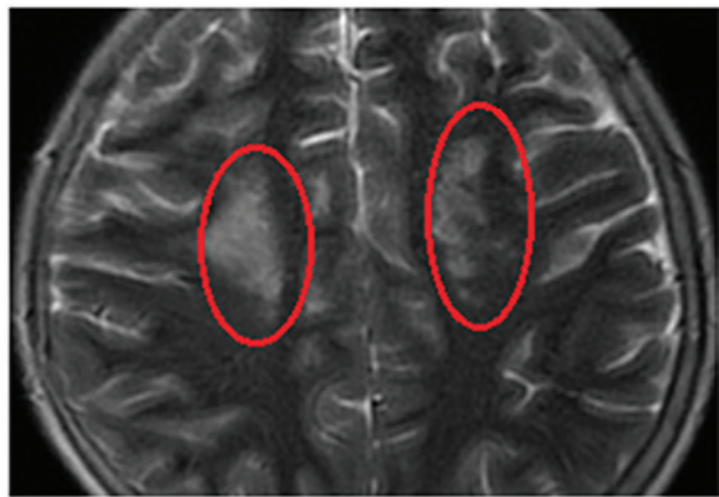

F

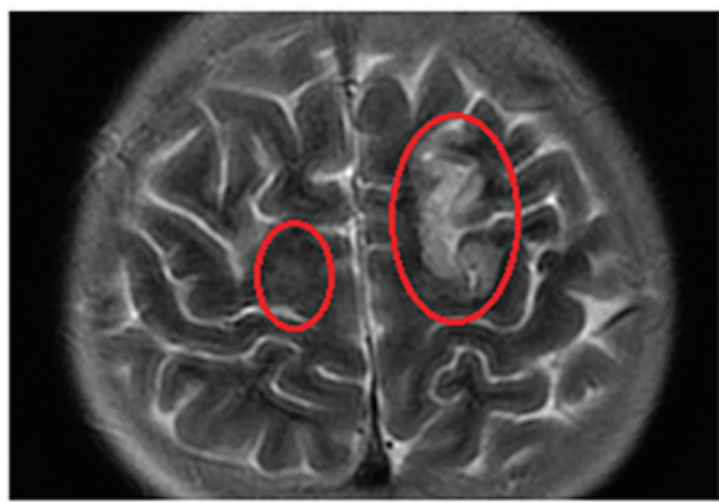

H

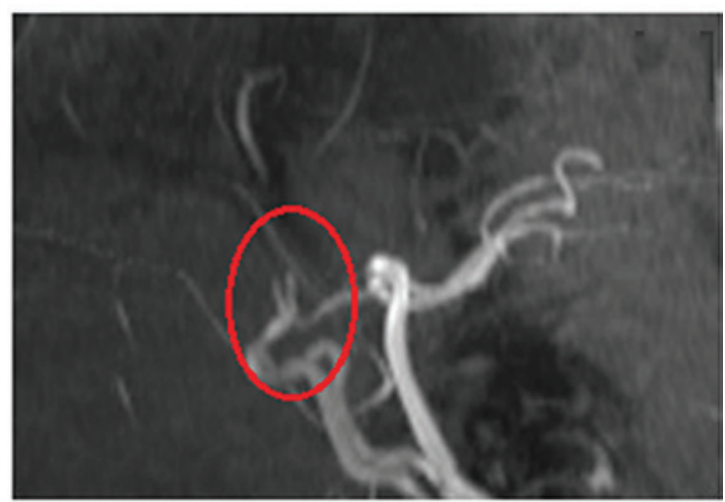

Figure 1. T2-weighted magnetic resonance images demonstrated multiple regions of cerebral infarction that involving the (A) bilateral basal ganglia, (B) white matter around the anterior horn of lateral ventricle, $(\mathrm{C})$ corona radiata, (D) centrum semiovale, (E) neurapophysis and (F) subcortical regions. Magnetic resonance angiography demonstrated serious stenosis at the bilateral terminal portion of the internal carotid arteries, and almost complete occlusion at the bilateral anterior and middle cerebral arteries. $(\mathrm{G})$ Horizontal and $(\mathrm{H})$ sagittal view. The red circles in the images indicate the area of interest.

by a case-control study conducted by Li et al, which demonstrated that increased thyroid function and elevated thyroid autoantibodies are associated with MMD, particularly in pediatric patients (10). Furthermore, improvement in the ischemic symptoms without recurrence has been reported following antithyroid drug administration alone in two patients affected by hyperthyroidism combined with bilateral internal carotid artery stenosis (21). In another study, two patients had 

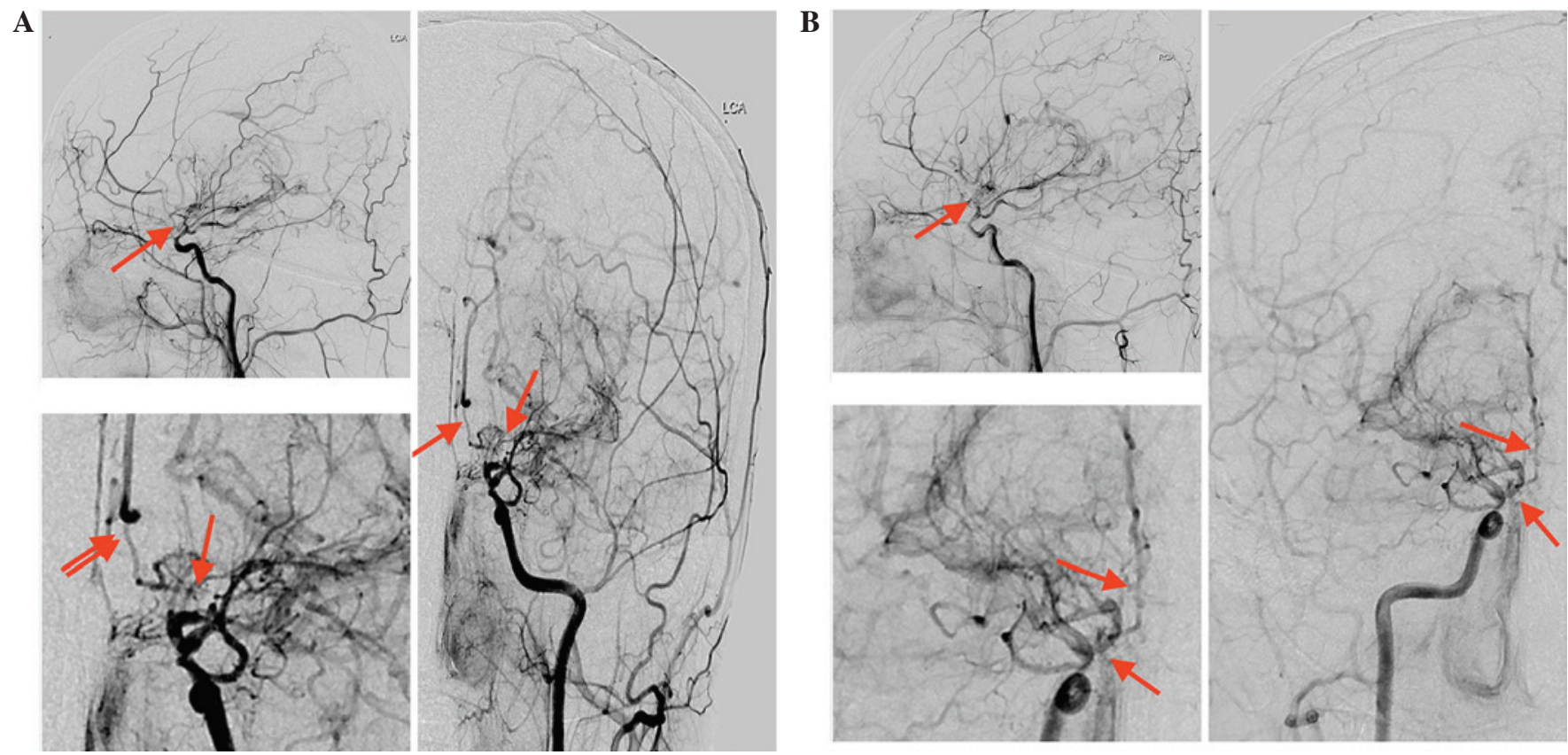

Figure 2. Digital subtraction angiography images of the (A) left internal carotid artery and (B) right internal carotid artery. The angiography images revealed severe stenosis of the bilateral distal segments of the internal carotid arteries, as well as the proximal segments of the anterior and middle cerebral arteries (indicates by the red arrows). Network collateral vessels were also observed in the bottom of the brain and bilateral basal ganglia area.

recurrent stroke subsequent to antithyroid drug withdrawal for 6 months, while the majority of patients in the same study did not present recurrence of stroke with regular use of antithyroid drugs (9). These studies indicate that GD is an underlying risk factor for MMD. However, the mechanism by which elevated thyroid autoantibodies or increased thyroid function evoke the development of stenosis of the intracranial arteries, as seen in MMD, remains to be elucidated.

The patient in the present study was diagnosed with MMD associated with GD, based on the symptoms of irritability, tremors, excessive sweating, frequent palpitations, the enlarged thyroid gland and the examination results of elevated TPO-Ab, elevated TG-Ab and positive TR-Ab in blood, along with thyroid gland pathology by needle biopsy. Furthermore, his multiple cerebral infarction and the results of DSA that showed a serious stenosis at the bilateral terminal portion of the ICAs, the bilateral MCAs and ACAs (Fig. 1). Notably, significant overactivation of FVIII and vWF were identified in the plasma on admission, which are considered to serve an important role in vascular diseases (15-18). The correlation of high plasma levels of vWF/FVIII with cardiovascular disease (15), cerebral sinus and deep venous thrombosis (18), as well as ischemic stroke $(16,17)$, has been extensively investigated. Certain studies have concluded that high plasma levels of vWF are predictive of ischemic stroke $(22,23)$, and may even be an independent risk factor for the prediction of ischemic stroke (23).

The pathophysiologic role of FVIII- or vWF-associated processes in ischemic brain injury is unclear. It is well-known that $\mathrm{vWF}$ and FVIII serve crucial roles in primary thrombosis. In addition, vWF mediates the initial adhesion of platelets at sites of vascular injury, and this subsequently leads to fibrin coagulation and the formation of a platelet thrombus via a complex coagulation cascade, platelets adhesion mediated by
vWF is a prerequisite for normal hemostasis (24-27). vWF is synthesized and stored in endothelial cells, and plasma levels are increased in response to different states of endothelial damage; therefore, $\mathrm{vWF}$ has been proposed as a useful marker of endothelial activation or dysfunction (14). Earlier studies have identified that concentric and eccentric fibrocellular thickening of the intima, which induce stenosis of the vascular lumen, are typical pathological changes in the terminal of the internal carotid artery in MMD $(28,29)$. Recent studies using high-resolution 3T MRI of a cross-section of the MCA showed no plaque or enhancement in the MCA wall $(9,30)$, The artery blood vessel image under 3T MRI from MMD is at variance with atherosclerotic features. This phenomenon was also observed in the patient of the present study. Furthermore, in another study, thromboemboli in the internal wall of the Moyamoya artery were observed in $\sim 50 \%$ of cases at autopsy (31). This indicates that abnormal thrombogenesis serves an important role in the etiology of MMD.

Previous studies have demonstrated that vascular endothelium is a specific target of thyroid hormones (32), and that hyperthyroidism tends to be associated with endothelial dysfunction or damage. This endothelial dysfunction depends not only on the cause but also on the degree of hyperthyroidism (19). Hyperthyroidism is known to result in high sympathetic nerve activity, thus plasma adrenaline levels are increased, acting as a strong stimulus of vWF secretion by endothelial cells (33). Clinical studies have also demonstrated that the elevated vWF levels in hyperthyroid patients returned to the normal range following administration of propranolol $(34,35)$. In accordance with the observations of the current study, hyperthyroidism has a significant correlation with the elevation of FVIII and vWF activity. Considering the pivotal role of vWF and FVIII in thrombogenesis, GD could be expected to be involved in the mechanism underlying the 
Table I. Examination results of thyroid function, coagulation parameters and thyroid autoantibodies on admission and during follow-up.

\begin{tabular}{|c|c|c|c|c|c|c|c|}
\hline Parameter & Reference range & Admission & $\begin{array}{c}\text { After } \\
3 \text { months }\end{array}$ & $\begin{array}{c}\text { After } \\
6 \text { months }\end{array}$ & $\begin{array}{c}\text { After } \\
9 \text { months }\end{array}$ & $\begin{array}{c}\text { After } \\
12 \text { months }\end{array}$ & $\begin{array}{c}\text { After } \\
18 \text { months }\end{array}$ \\
\hline TT3 (nmol/l) & $0.89-2.44$ & 7.04 & 0.97 & 3.48 & 1.17 & 4.39 & 1.85 \\
\hline TT4 (nmol/l) & $62.68-150.84$ & 210.73 & 64.06 & 171.65 & 52.12 & 220.58 & 113.50 \\
\hline $\mathrm{TSH}(\mu \mathrm{U} / \mathrm{ml})$ & $0.35-4.94$ & $<0.001$ & 0.027 & 0.001 & 3.803 & 0.048 & 0.007 \\
\hline FT3 (pmol/l) & $2.63-5.70$ & 29.17 & 3.21 & 10.63 & 3.26 & 15.32 & 5.45 \\
\hline FT4 (pmol/l) & $9-19.04$ & 35.99 & 11.31 & 27.63 & 10.26 & 34.16 & 19.06 \\
\hline FVIII (\%) & $50-150$ & 261.60 & 122.40 & 175.90 & 115.60 & 185.60 & 151.80 \\
\hline vWF (\%) & $40-120$ & 324.20 & 117.10 & 128.00 & 119.50 & 146.50 & 125.40 \\
\hline FVII (\%) & $50-150$ & 125.00 & 75.00 & 113.00 & 55.00 & 59.00 & 60.0 \\
\hline FIX (\%) & $50-150$ & 148.20 & 138.60 & 137.20 & 129.70 & 115.80 & 66.0 \\
\hline PT-S (sec) & $9.4-12.5$ & 12.60 & 11.30 & 11.90 & 11.50 & 9.68 & 10.80 \\
\hline PT (\%) & $70-120$ & 100.70 & 80.00 & 89.00 & 112.00 & 108.00 & 94.00 \\
\hline PT-INR & $0.9-1.2$ & 1.04 & 1.09 & 1.14 & 0.98 & 1.05 & 1.01 \\
\hline FIB-C (mg/l) & $200-400$ & 251 & 230 & 381 & 418 & 352 & 228 \\
\hline APTT (sec) & $25.5-38.4$ & 24.70 & 25.50 & 27.80 & 24.50 & 29.50 & 29.20 \\
\hline APTT-R & $0.91-1.38$ & 0.89 & 0.89 & 0.98 & 0.93 & 1.12 & 1.02 \\
\hline TPO-Ab (U/ml) & $0-12$ & 396.44 & 205.17 & 111.21 & 221.3 & 252.1 & 594.9 \\
\hline TG-Ab (U/ml) & $0-34$ & 134.56 & 71.31 & 65.63 & 85.20 & 74.50 & 255.30 \\
\hline $\mathrm{TR}-\mathrm{Ab}$ & - & + & + & $+/-$ & + & + & + \\
\hline
\end{tabular}

TT3: triiodothyronine; TT4: thyroxin; TSH: thyroid-stimulating hormone; FT3: free triiodothyronine; FT4: free thyroxin; FVIII: blood coagulation factor FVIII activity; vWF: von Willebrand factor activity; FVII: blood coagulation factor VII activity; FIX: blood coagulation factor IX activity; PT-S: prothrombin time, PT: prothrombin activity, PT-INR: prothrombin international normalized ratio, FIB: fiber fibrinogen, APTT: activated partial thromboplastin time, APTT-R: partial thromboplastin time ratio of activate; TR-Ab: thyroid hormone receptor antibody; TPO-Ab: thyroid peroxidase antibody; TG-Ab: thyroglobulin antibody.

development of MMD, which is mediated by vWF/FVIII overactivation. GD is such a complex disease that may influence multiple aspects associated with the pathophysiologic process of MMD, including cerebral hemodynamic changes (9), autoimmune inflammation $(10,11)$ and endothelial dysfunction (19). In addition, thrombogenesis is mediated by vWF and FVIII activities, which may be due to injury to the intima or vessel wall, while the injury of intima or vessel wall could be a result of cerebral hemodynamic changes or autoimmune inflammation. Further evidence of vWF and FVIII mediating the development of MMD is the varying trend of vWF and FVIII activities during the follow-up period in the present study. Upon admission, the current patient had significantly elevated vWF and FVIII activities, and presented with rapid neurological deterioration. Following the administration of thiamazole and metoprolol, the patient's thyroid function returned to normal, while vWF and FVIII activities were simultaneously suppressed, with a correlation observed in their fluctuations (Table I). During the follow-up period, the thyroid autoantibodies TR-Ab, TG-Ab and TPO-Ab persisted at high levels in the patient's blood, whereas other coagulation parameters were consistently within or close to the normal range. Therefore, the effect of thyroid autoantibodies on the development of MMD was unclear in the present patient.

However, an evident limitation of the present study is that only one case was included. Nevertheless, during the 20-month follow-up period, correlations were identified among GD, overactivation of vWF/FVIII and intracranial arterial stenosis, which require further confirmation in more cases and in-depth studies in the future. Moyamoya disease associated Graves' disease has a tendency to present with coagulation dysfunction. Overactivation of $\mathrm{vWF} / \mathrm{FVIII}$ due to thyrotoxicosis might contribute to the ischemic accidents. Future research is warranted to investigate novel therapeutic methods in prevention of ischemic attack.

\section{Acknowledgements}

The present study was supported by a grant from the National Youth Science Fund (grant no. 81301118). The authors thank Dr Rong Wang and Dr Wei-Qing Wan and their colleagues (Center of Cerebrovascular Disease, Beijing Tian Tan Hospital, Capital Medical University, Beijing, China) for their excellent vascular surgical procedures on the patient. In addition, we also thank the patient and his parents for their best collaboration during the follow-up.

\section{References}

1. Houkin K, Ito M, Sugiyama T, Shichonohe H, Nakayama N, Kazumata K and Kuroda S: Review of past research and current concepts on the etiology of moyamoya disease. Neurol Med Chir (Tokyo) 52: 267-277, 2012. 
2. Kudo T: Spontaneous occlusion of the circle of Willis. A disease apparently confined to Japanese. Neurology 18: 485-496, 1968.

3. Suzuki J and Kodama N: Cerebrovascular "Moyamoya" disease. 2. Collateral routes to forebrain via ethmoid sinus and superior nasal meatus. Angiology 22: 223-236, 1971.

4. Chen PC, Yang SH, Chien KL, Tsai IJ and Kuo MF: Epidemiology of moyamoya disease in Taiwan: A nationwide population-based study. Stroke 45: 1258-1263, 2014.

5. Kushima K, Satoh Y, Ban Y, Taniyama M, Ito K and Sugita K Graves' thyrotoxicosis and Moyamoya disease. Can J Neurol Sci 18: 140-142, 1991.

6. Ohba S, Nakagawa T and Murakami H: Concurrent Graves' disease and intracranial arterial stenosis/occlusion: special considerations regarding the state of thyroid function, etiology, and treatment. Neurosurg Rev 34: 297-304, 2011.

7. Hsu SW, Chaloupka JC and Fattal D: Rapidly progressive fatal bihemispheric infarction secondary to Moyamoya syndrome in association with Graves thyrotoxicosis. AJNR Am J Neuroradiol 27: 643-647, 2006

8. Inaba M, Henmi Y, Kumeda Y, Ueda M, Nagata M, Emoto M, Ishikawa T, Ishimura E and Nishizawa Y: Increased stiffness in common carotid artery in hyperthyroid Graves' disease patients. Biomed Pharmacother 56: 241-246, 2002.

9. Ni J, Zhou LX, Wei YP, Li ML, Xu WH, Gao S and Cui LY: Moyamoya syndrome associated with Graves' disease: A case series study. Ann Transl Med 2: 77, 2014.

10. Li H, Zhang ZS, Dong ZN, Ma MJ, Yang WZ, Han C, Du MM Liu YX, Yang H, Liu W, et al: Increased thyroid function and elevated thyroid autoantibodies in pediatric patients with moyamoya disease: A case-control study. Stroke 42: 1138-1139, 2011.

11. Kim SJ, Heo KG, Shin HY, Bang OY, Kim GM, Chung CS, Kim KH, Jeon P, Kim JS, Hong SC and Lee KH: Association of thyroid autoantibodies with moyamoya-type cerebrovascular disease: A prospective study. Stroke 41: 173-176, 2010.

12. Panegyres PK, Morris JG, O'Neill PJ and Balleine R: Moyamoya-like disease with inflammation. Eur Neurol 33 260-263, 1993

13. Tendler BE, Shoukri K, Malchoff C, MacGillivray D, Duckrow R, Talmadge T and Ramsby GR: Concurrence of Graves' disease and dysplastic cerebral blood vessels of the moyamoya variety. Thyroid 7: 625-629, 1997.

14. Lip GY and Blann A: von Willebrand factor: A marker of endothelial dysfunction in vascular disorders? Cardiovasc Res 34 255-265, 1997.

15. Vischer UM: von Willebrand factor, endothelial dysfunction, and cardiovascular disease. J Thromb Haemost 4: 1186-1193, 2006

16. Samai A, Monlezun D, Shaban A, George A, Dowell L, Kruse-Jarres R, Schluter L, El Khoury R and Martin-Schild S: Von Willebrand factor drives the association between elevated factor VIII and poor outcomes in patients with ischemic stroke. Stroke 45: 2789-2791, 2014

17. Wieberdink RG, van Schie MC, Koudstaal PJ, Hofman A, Witteman JC, de Maat MP, Leebeek FW and Breteler MM: High von Willebrand factor levels increase the risk of stroke: The Rotterdam study. Stroke 41: 2151-2156, 2010.

18. Koster T, Blann AD, Briët E, Vandenbroucke JP and Rosendaal FR: Role of clotting factor VIII in effect of von Willebrand factor on occurrence of deep-vein thrombosis. Lancet 345: 152-155, 1995.
19. Popławska-Kita A, Szelachowska M, Modzelewska A, Siewko K, Dzięcioł J, Klimiuk PA and Górska M: Endothelial dysfunction in Graves' disease. Adv Med Sci 58: 31-37, 2013.

20. Research Committee on the Pathology and Treatment of Spontaneous Occlusion of the Circle of Willis; Health Labour Sciences Research Grant for Research on Measures for Infractable Diseases: Guidelines for diagnosis and treatment of moyamoya disease (spontaneous occlusion of the circle of Willis). Neurol Med Chir (Tokyo) 52: 245-266, 2012.

21. Kamasaki H, Takeuchi T,Mikami T, Komeichi K and Tsutsumi H: A case of graves' disease diagnosed in the course of bilateral carotid artery stenoses (moyamoya disease); a case report and review of the literature. Clin Pediatr Endocrinol 22: 39-44, 2013

22. Conway DS, Pearce LA, Chin BS, Hart RG and Lip GY: Prognostic value of plasma von Willebrand factor and soluble P-selectin as indices of endothelial damage and platelet activation in 994 patients with nonvalvular atrial fibrillation. Circulation 107: 3141-3145, 2003

23. Carter AM, Catto AJ, Mansfield MW, Bamford JM and Grant PJ: Predictive variables for mortality after acute ischemic stroke. Stroke 38: 1873-1880, 2007.

24. Sadler JE: Biochemistry and genetics of von Willebrand factor. Annu Rev Biochem 67: 395-424, 1998.

25. Lenting PJ, Casari C, Christophe OD and Denis CV: von Willebrand factor: The old, the new and the unknown. J ThrombHaemost 10: 2428-2437, 2012.

26. Müller G: [Structure and function of the factor VIII/von Willebrand factor complex]. Z Gesamte Inn Med 45: 65-68, 1990 (In German).

27. Hoyer LW: The factor VIII complex: Structure and function. Blood 58: 1-13, 1981.

28. Rao M, Zhang H, Liu Q, Zhang S, Hu L and Deng F: Clinical and experimental pathology of Moyamoya disease. Chin Med J (Engl) 116: 1845-1849, 2003.

29. Ikeda $E$ and Hosoda Y: Distribution of thrombotic lesions in the cerebral arteries in spontaneous occlusion of the circle of Willis: Cerebrovascular moyamoya disease. Clin Neuropathol 12: 44-48, 1993.

30. Kim YJ, Lee DH, Kwon JY, Kang DW, Suh DC, Kim JS and Kwon SU: High resolution MRI difference between moyamoya disease and intracranial atherosclerosis. Eur J Neurol 20: 1311-1318, 2013

31. Hosoda Y, Ikeda E and Hirose S: Histopathological studies on spontaneous occlusion of the circle of Willis (cerebrovascular moyamoya disease). Clin Neurol Neurosurg 99 (Suppl 2): S203-S208, 1997.

32. Napoli R, Biondi B, Guardasole V, Matarazzo M, Pardo F, Angelini V, Fazio S and Saccà L: Impact of hyperthyroidism and its correction on vascular reactivity in humans. Circulation 104: 3076-3080, 2001.

33. Vischer UM, Lang U and Wollheim CB: Autocrine regulation of endothelial exocytosis: Von Willebrand factor release is induced by prostacyclin in cultured endothelial cells. FEBS Lett 424 : 211-215, 1998.

34. Liu L, Wang X, Lin Z and Wu H: Elevated plasma levels of VWF: $\mathrm{Ag}$ in hyperthyroidism are mediated through beta-adrenergic receptors. Endocr Res 19: 123-133, 1993.

35. Burggraaf J, Lalezari S, Emeis JJ, Vischer UM, de Meyer PH, Pijl $\mathrm{H}$ and Cohen AF: Endothelial function in patients with hyperthyroidism before and after treatment with propranolol and thiamazol. Thyroid 11: 153-160, 2001. 